\title{
CT findings of high-attenuation pulmonary abnormalities
}

\author{
Naim Ceylan • Selen Bayraktaroglu • Recep Savaș • \\ Hudaver Alper
}

Received: 5 June 2010 /Revised: 10 July 2010 / Accepted: 16 August 2010 / Published online: 4 September 2010

(C) European Society of Radiology 2010

\begin{abstract}
Objectives To review the computed tomography (CT) findings of common and uncommon high-attenuation pulmonary lesions and to present a classification scheme of the various entities that can result in high-attenuation pulmonary abnormalities based on the pattern and distribution of findings on $\mathrm{CT}$.

Background High-attenuation pulmonary abnormalities can result from the deposition of calcium or, less commonly, other high-attenuation material such as talc, amiodarone, iron, tin, mercury and barium sulphate. CT is highly sensitive in the detection of areas of abnormally high attenuation in the lung parenchyma, airways, mediastinum and pleura. The cause of the calcifications and other highattenuation conditions may be determined based on the location and pattern of the abnormalities within the lung parenchyma and knowledge of the associated clinical features.

Results We have presented a diagnostic approach based on the presence and distribution of five main patterns of highattenuation conditions on CT: (1) small hyperdense nodules, (2) large calcified nodules or masses, (3) high-attenuation linear or reticular pattern, (4) high-attenuation consolidation and (5) high attenuation extraparenchymal lesions.

Conclusions Some high-attenuation pulmonary abnormalities have characteristic CT findings suggesting the correct diagnosis. In other diseases, a combination of clinical
\end{abstract}

N. Ceylan $(\varangle) \cdot$ S. Bayraktaroglu $\cdot$ R. Savaș $\cdot$ H. Alper Department of Radiology, Ege University School of Medicine, Bornova, Izmir 35100, Turkey

e-mail: ceylannaim@gmail.com features and radiological findings can significantly improve diagnostic accuracy.

Keywords Computed tomography $\cdot$ Pulmonary abnormalities $\cdot$ High attenuation $\cdot$ Nodules $\cdot$ Reticular pattern $\cdot$ Consolidation $\cdot$ Extraparenchymal lesions

\section{Introduction}

High-attenuation pulmonary abnormalities can result from a variety of different conditions, including from the deposition of calcium. Amiodarone pulmonary toxicity may cause high-attenuation pulmonary parenchymal opacities. Multiple dense nodular opacities are rarely seen in siderosis, stannosis, talcosis and baritosis, in which iron, tin, talc and barium sulfate respectively are deposited in the lungs. Computed tomography (CT) is highly sensitive in the detection of areas of abnormally high attenuation in the lung parenchyma, airways, mediastinum and pleura. Calcifications in the thorax are frequently manifestations of previous infectious processes. However, they may be due to benign or malign neoplasms, metabolic disorders, or occupational exposure. The cause of the calcifications and other high-attenuation conditions may be determined by means of the location and pattern of the abnormalities within the lung parenchyma and knowledge of the associated clinical features. High-attenuation pulmonary abnormalities can be divided into five main patterns on CT: small hyperdense nodules, large nodules or masses, highattenuation linear or reticular pattern, high-attenuation consolidation, and high-attenuation extraparenchymal lesions. 


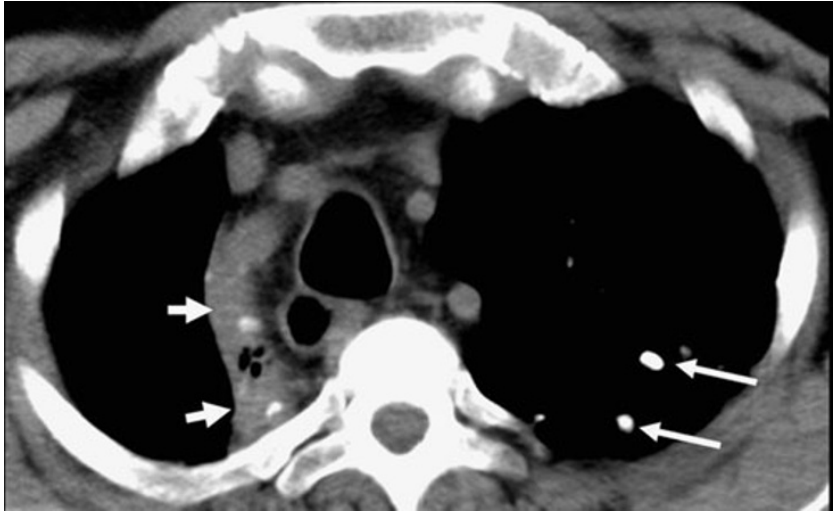

Fig. 1 Residual postprimary pulmonary tuberculosis. Axial CT shows calcified granulomatous nodules in the left upper lobe (long arrows). Calcified nodules and the sequelae of parenchymal changes are also seen in the right upper lobe (short arrows)

\section{Small hyperdense nodules}

Small hyperdense nodules are nodular opacities measuring less than $10 \mathrm{~mm}$ in diameter, showing focal or diffuse distribution in the lung parenchyma. Small hyperdense nodules can be secondary to dystrophic calcification in

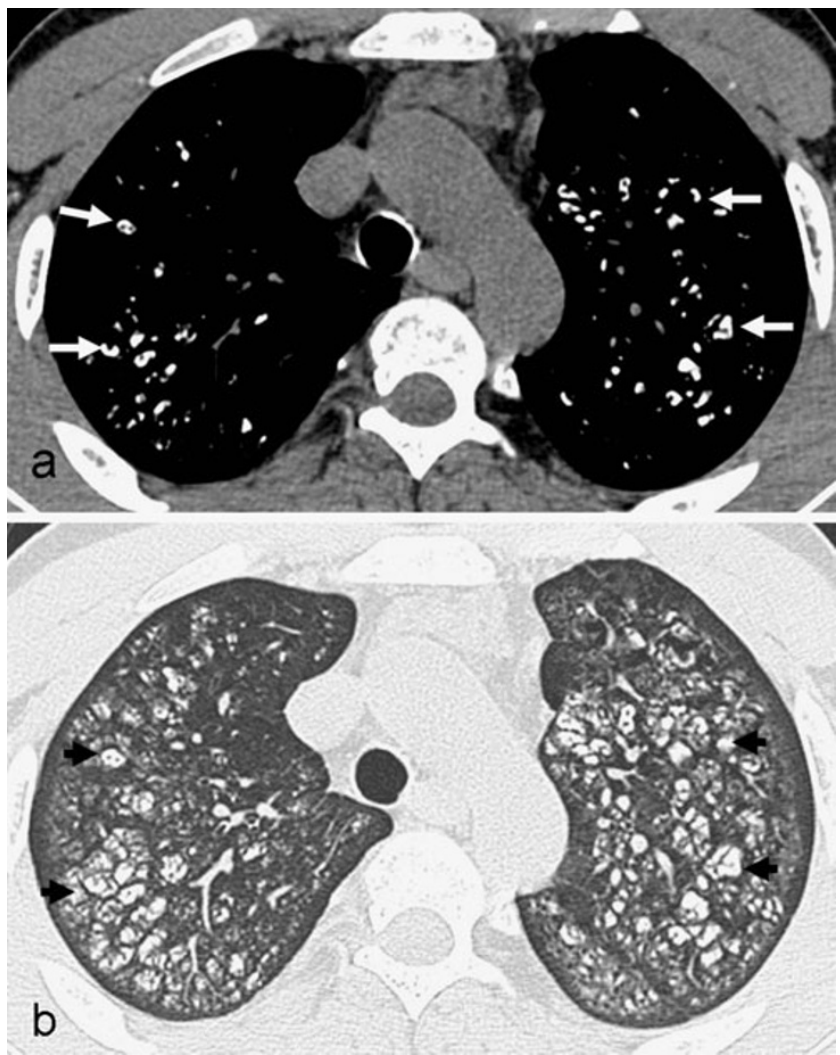

Fig. 2 Metastatic pulmonary calcification in a 24-year-old man who had known chronic renal failure. Axial CT at a mediastinal and b parenchymal windows demonstrates bilateral centrilobular fluffy ground-glass nodular opacities that contain foci of calcification (arrows)

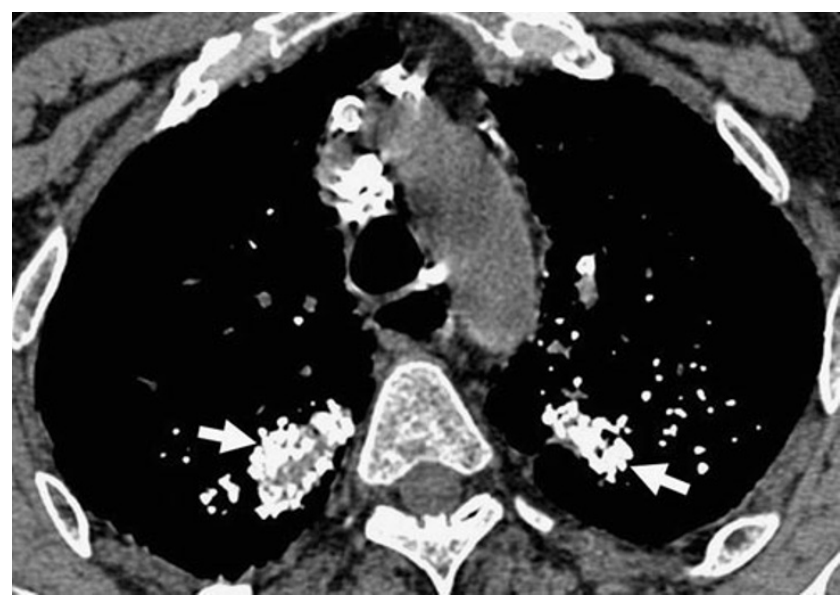

Fig. 3 Silicosis. Axial CT at the mediastinal window shows multiple calcified nodules with a conglomerate mass of fibrosis in the upper lobes (arrows)

previously damaged lung parenchyma. Small calcified parenchymal nodules most commonly are a result of infectious diseases. Other causes of small hyperdense nodules are pulmonary metastases, chronic haemorrhagic conditions, occupational diseases, deposition diseases, talcosis and idiopathic disorders such as pulmonary alveolar microlithiasis [1-3].
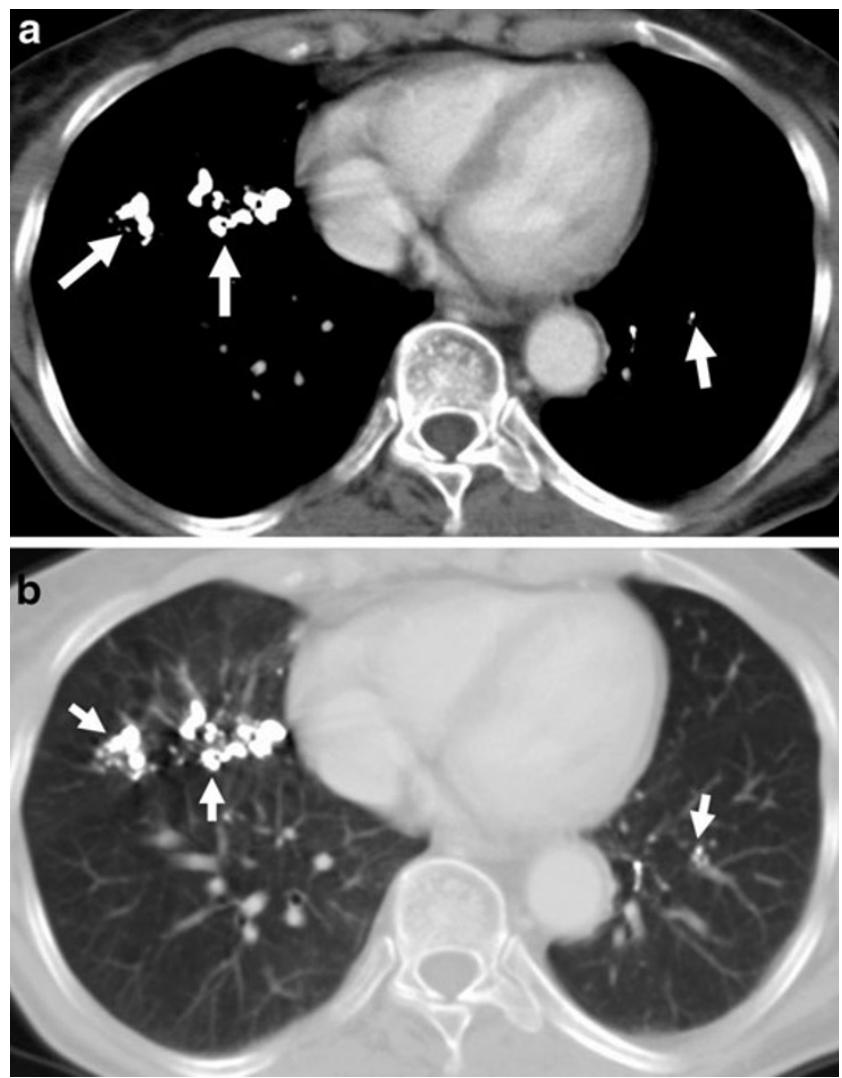

Fig. 4 Barium aspiration. Axial CT at a mediastinal and b parenchymal windows shows extremely dense opacities in both lower lobes (arrows) 

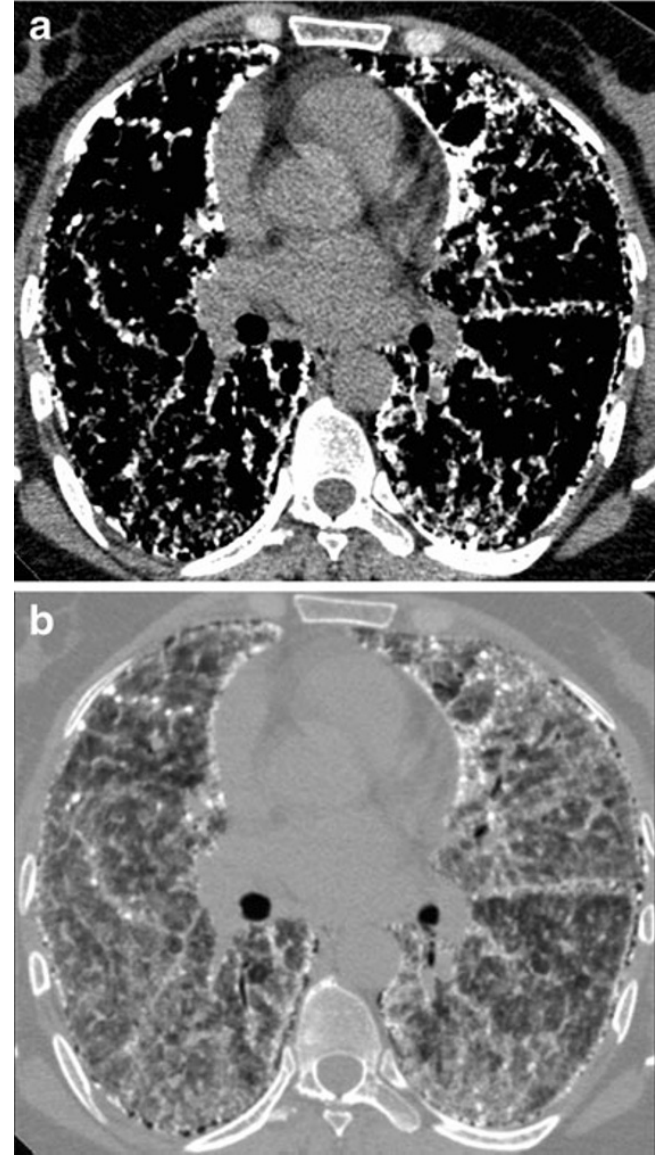

Fig. 5 Pulmonary alveolar microlithiasis. Axial CT at a mediastinal and $\mathbf{b}$ parenchymal windows shows diffuse numerous dense micronodules with calcified thickening of interlobular septa and subpleural cysts

Calcified parenchymal nodules are frequently seen in tuberculosis. The sequela dystrophic calcification follows caseation, necrosis or fibrosis. These nodules are seen as well circumscribed parenchymal calcifications with fibrosis on CT (Fig. 1). Most patients with pulmonary nodular

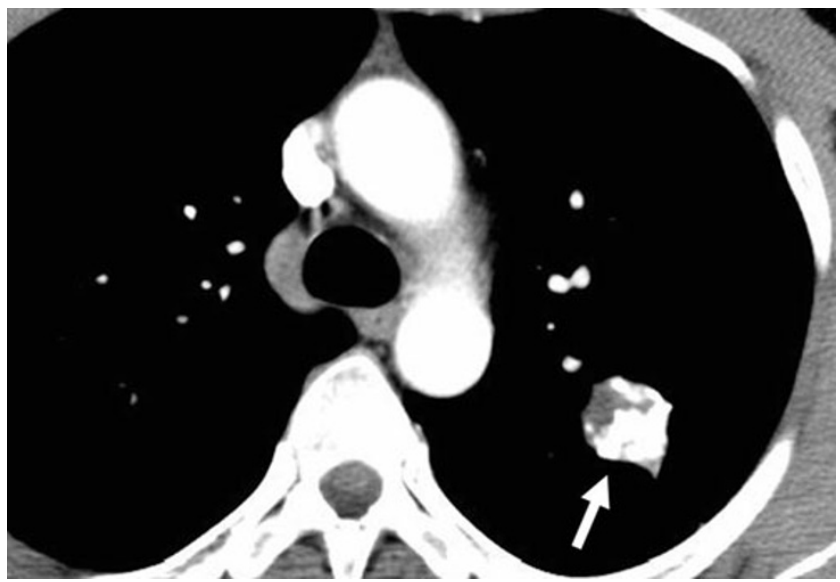

Fig. 6 Hamartomas. Axial CT shows popcorn calcification in a benign solitary pulmonary nodule (arrow)

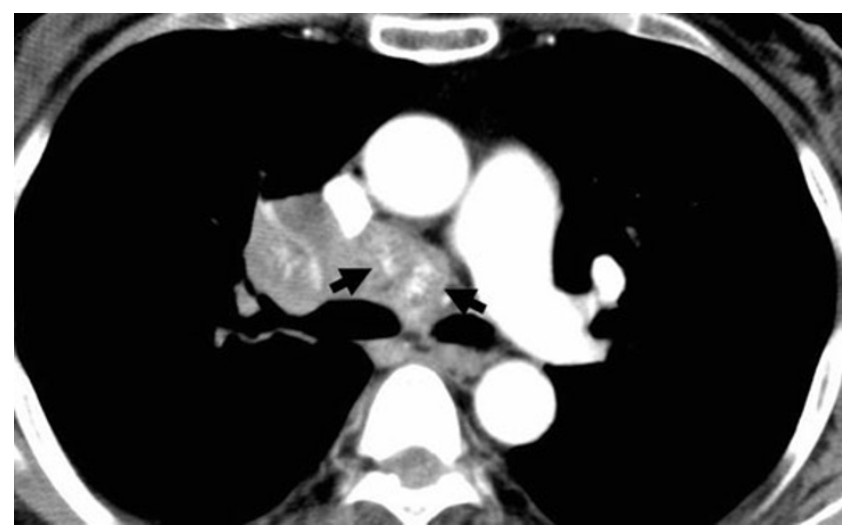

Fig. 7 Lung carcinoma. Axial CT shows eccentric calcification in a malignant mass, invading the mediastinum and right hilar region (arrows)

calcifications secondary to tuberculosis have calcified hilar or mediastinal lymph nodes, known together as the Ranke complex. Histoplasmosis and varicella infections may less commonly lead to parenchymal calcified nodules [1]. Widespread micronodular calcification can be seen in the late period of varicella infection.

Metastatic pulmonary calcification is a consequence of calcium deposition in normal pulmonary parenchyma. This condition can occur in a variety of benign and malignant disorders such as primary and secondary hyperparathyroidism, chronic renal failure, sarcoidosis, vitamin D intoxication, IV calcium therapy, multiple myeloma and massive osteolysis caused by metastases [4]. High-resolution CT (HRCT) findings are characterised by centrilobular fluffy ground-glass nodular opacities that contain foci of calcification (Fig. 2). Metastatic pulmonary calcification is typically most marked in the upper lobes.

Idiopathic pulmonary haemosiderosis is an uncommon cause of alveolar haemorrhage that occurs predominantly in infants and young adults. This disorder is characterised by

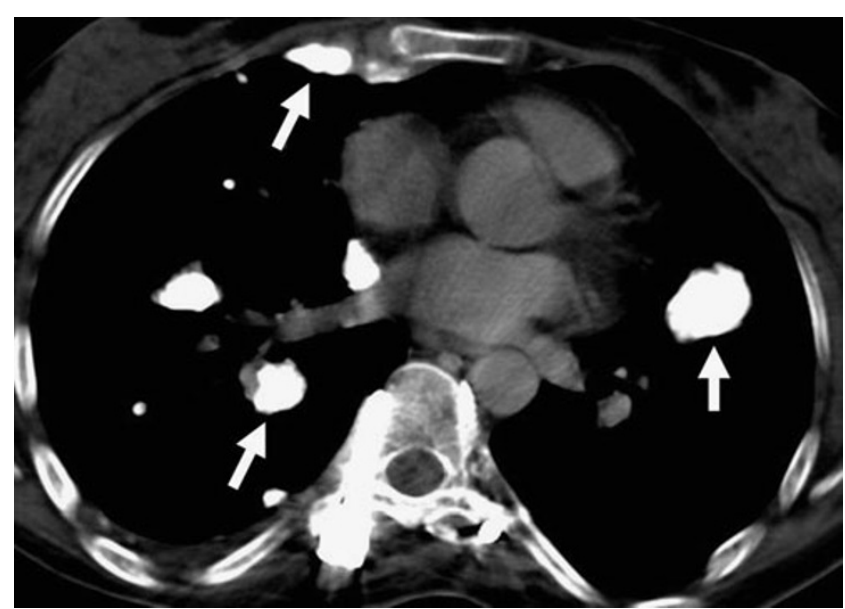

Fig. 8 Metastatic osteosarcoma. Axial CT shows parenchymal and pleural calcified metastatic lesions in both haemothoraces (arrows) 


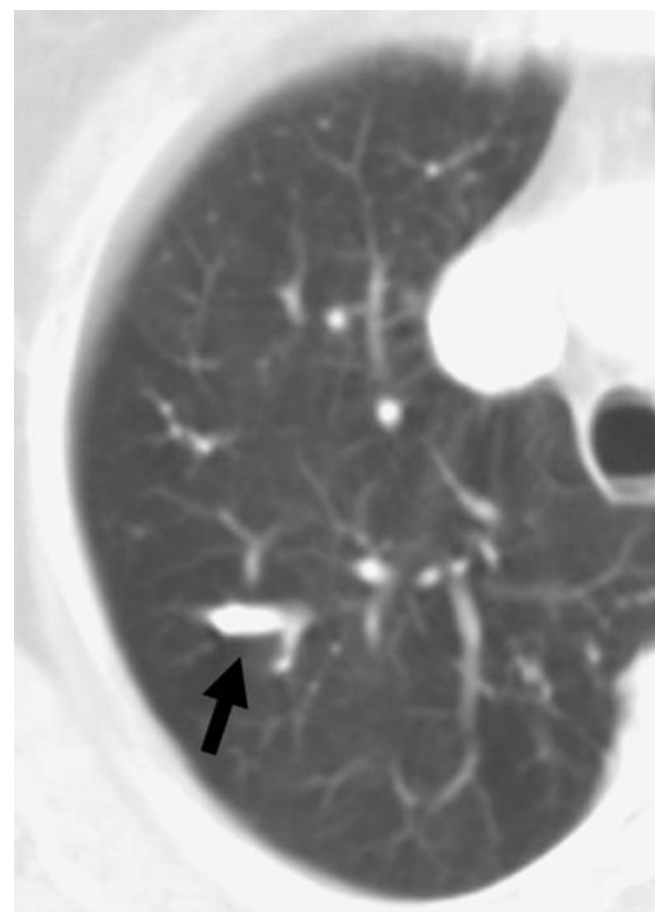

Fig. 9 Mucus plugging. Axial CT (lung window) shows calcified linear bronchial opacity in the right upper lobe (arrow)

recurrent episodes of alveolar haemorrhage. HRCT shows dense centrilobular nodular opacities due to recurrent haemorrhage. Secondary haemosiderosis due to mitral stenosis may present with small calcified nodules.

Diffuse small calcified nodules, often associated with egg-shell calcification of hilar or mediastinal lymph nodes, can occur in silicosis and coal workers' pneumoconiosis. Silicosis is caused by inhalation of free silica particles, usually during occupational exposure such as mining, sandblasting and masonry. Radiographic evidence of silicosis typically develops after 10-20 years of exposure to low concentrations of silica dust [5]. HRCT findings of silicosis include diffuse and randomly distributed small well-defined nodules that are most prominent in the upper lobes [6]. These calcified nodules are commonly seen with massive fibrosis (Fig. 3).

Multiple dense nodular opacities are rarely seen in siderosis, stannosis and baritosis, in which iron, tin and barium respectively are deposited in the lungs. In siderosis, nodular opacities are less dense and less profuse than those in silicosis. HRCT shows extremely dense opacities due to barium aspiration in baritosis, usually locating in the basal segments of the lower lobes (Fig. 4).

Alveolar microlithiasis, a rare disease of unknown origin, is characterized by diffuse sand-like calcifications within the alveoli. This disorder may be detected incidentally on chest radiographs obtained for other reasons. Characteristic HRCT findings consist of multiple innumerable
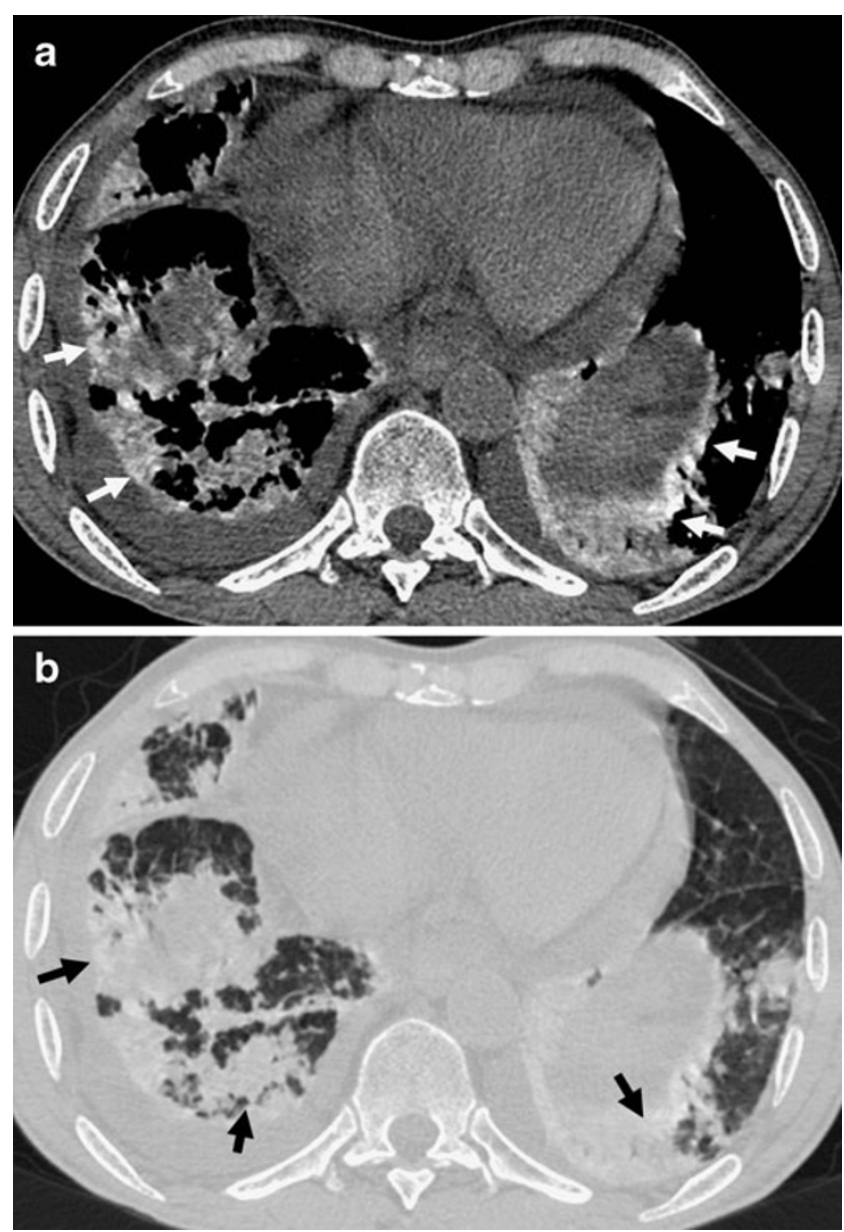

Fig. 10 Amiodarone toxicity. Axial unenhanced CT at a mediastinal and $\mathbf{b}$ parenchymal windows shows dense lung consolidations in both lower lobes (arrows). Bilateral pleural effusion and pericardial effusion are also seen

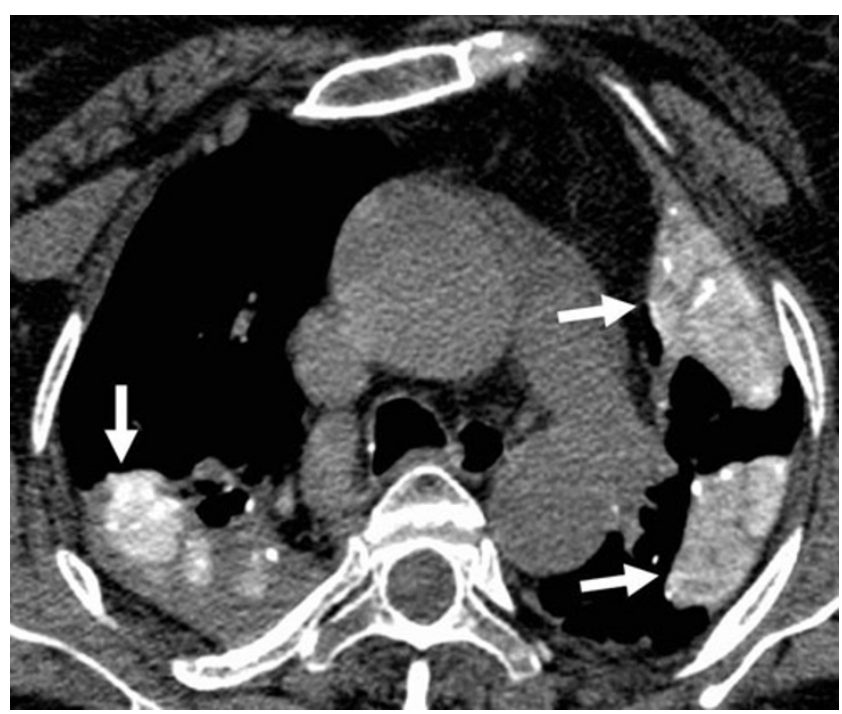

Fig. 11 Calcified atelectasis. Axial unenhanced CT at the mediastinal window shows high-attenuation consolidations in both upper lobes (arrows) 


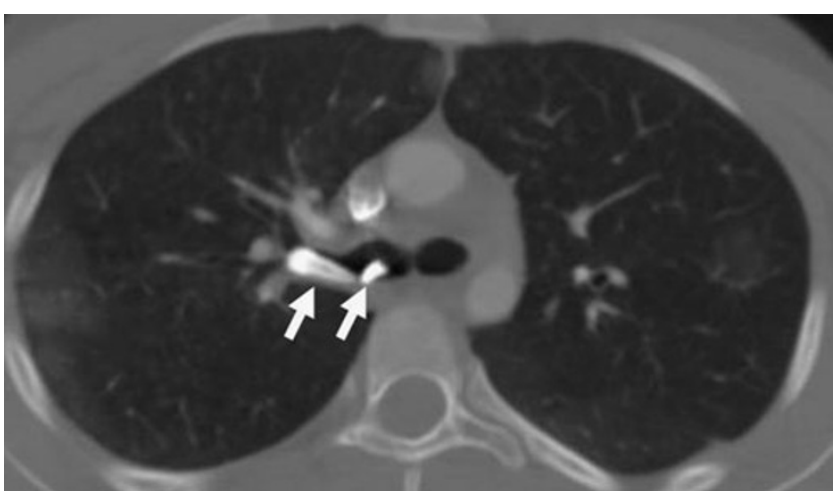

Fig. 12 Endobronchial teeth secondary to trauma. Axial CT (bone window) demonstrates teeth in the right main and upper lobe bronchus (arrows)

tiny sand-like calcified micronodules that tendency toward confluence throughout both lungs. Other findings include calcified interlobular septa and small subpleural cysts. Another feature seen on HRCT includes a very low attenuation line alongside the pleura, called the "black pleural line" (Fig. 5).

Talcosis is seen in workers exposed to talc during extraction of magnesium silicate from mines and grinding. Another form of talcosis can be seen in drug users who inject talc. When dissolved and injected intravenously, talc particles become deposited within pulmonary arterioles, capillaries and interstitium.

HRCT findings consist of numerous high-attenuation well-defined micronodules or diffuse ground-glass opacities. Over time the nodules tend to confluence, resulting in high-attenuation confluent masses.

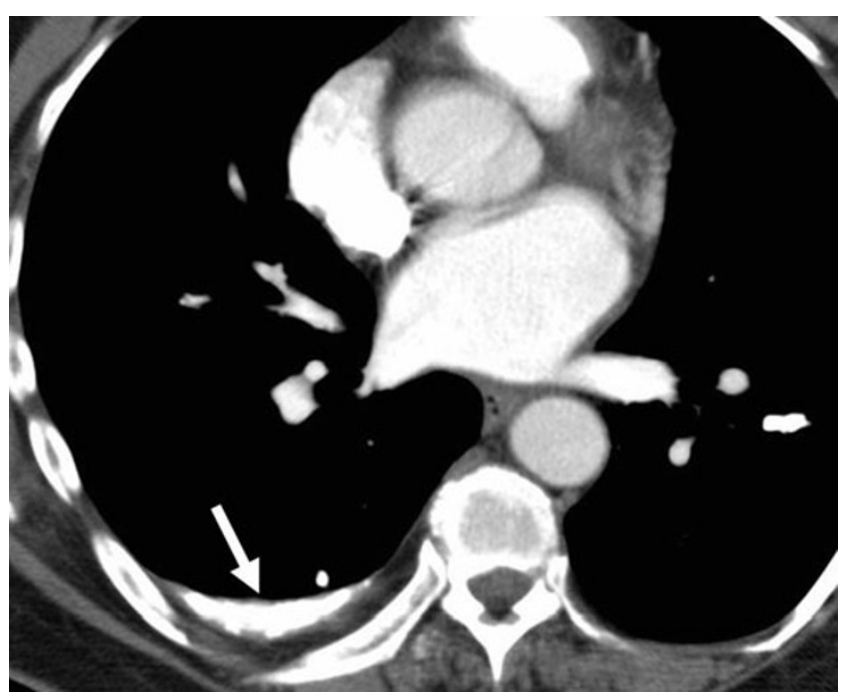

Fig. 13 Pleural calcification secondary to empyema. Axial CT shows pleural calcification at the right haemothorax (arrow)

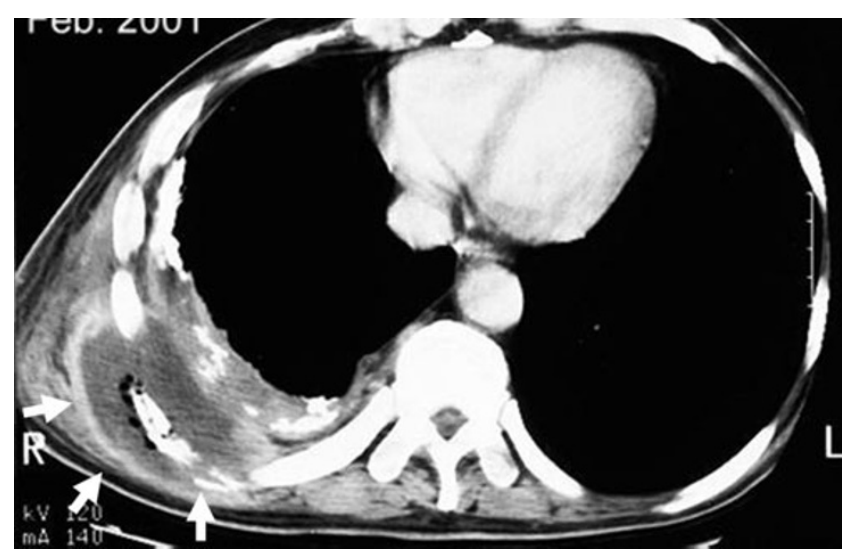

Fig. 14 Empyema necessitans after tuberculous empyema. Axial CT shows a calcified pleural mass, extending the extrapleural structures at the right haemothorax (arrows)

\section{Large calcified nodules or masses}

Calcification can be seen in a variety of benign and malignant tumours such as hamartomas, chondromas, plasmocytomas, carcinoid tumours and bronchial carcinomas [7]. Hamartoma is the most common benign tumour of the lung. Calcification can be detected on CT in over $30 \%$ of cases. Hamartomas have characteristic popcorn-like or diffuse calcification (Fig. 6). Bronchogenic carcinoma may rarely contain calcification, usually eccentric in location (Fig. 7). Calcification within tumours occurs by three mechanisms: (1) calcified scar tissue or granulomatous disease engulfed by tumour, (2) dystrophic calcification within areas of tumour necrosis and (3) calcium deposition within the tumour as a result of the secretory function of the carcinoma itself.

Calcification in pulmonary metastases is rare and can result from either sarcomas (osteosarcomas, chondrosarcomas and synovial sarcomas) or carcinomas (mucin-producing carcinomas, thyroid carcinomas and treated metastatic choriocarcinomas). Osteosarcoma may lead to multiple, calcified parenchymal and pleural metastases (Fig. 8). Bone

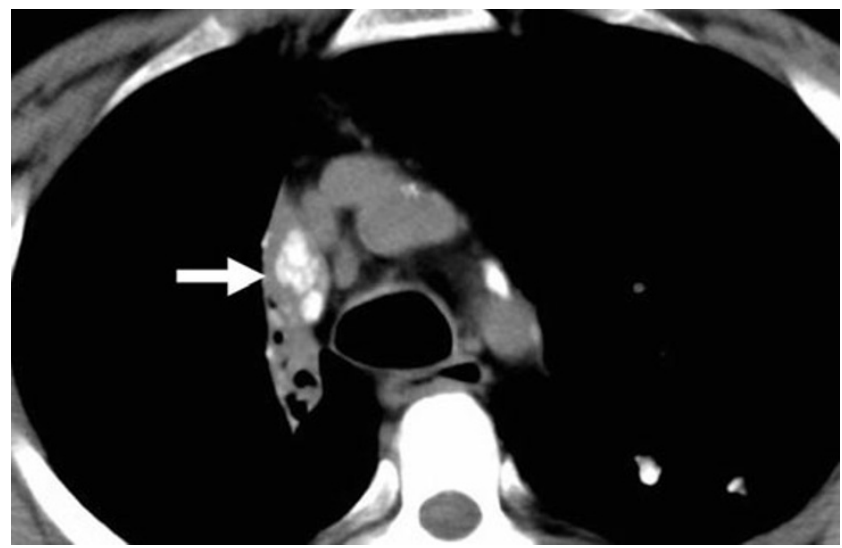

Fig. 15 Lymph node calcification. Axial CT shows right paratracheal calcified lymph nodes secondary to tuberculosis (arrow) 
formation in osteoid tumour, calcification and ossification of tumour cartilage, dystrophic calcification and mucoid calcification are mechanisms responsible for calcification in metastases.

Parenchymal calcified nodules or consolidation may be seen in pulmonary amyloidosis.

\section{High-attenuation linear or reticular pattern}

Diffuse pulmonary ossification is an uncommon condition that is characterised by metaplastic bone formation in the lung parenchyma. It is associated with a variety of pulmonary, cardiac and systemic disorders. It may be localised or distributed widely [8].

Linear calcification through the lung interstitium is occasionally seen in idiopathic pulmonary fibrosis.

Long-standing mucus plugging may progress to linear or nodular calcification (Fig. 9).

\section{High-attenuation consolidation}

Deposition of iodine can occur within the lung parenchyma as a result of treatment with amiodarone, a tri-iodinated anti-arrhythmic drug. Pulmonary toxicity occurs in $2-18 \%$ of patients [3]. The most common CT findings include septal thickening, interstitial fibrosis and high attenuation consolidations (Fig. 10). The association of dense lung airspace consolidations with a high density of the liver and spleen is characteristic of amiodarone exposure.

Chronic atelectasis can be rarely seen as calcified consolidation (Fig. 11).

\section{High-attenuation extraparenchymal lesions}

Aspiration of teeth or dental fragments may be seen after trauma. CT shows high-attenuation endobronchial lesions (Fig. 12).

Pleural calcification occurs most often as the result of long-standing inflammatory diseases, such as empyema, haemothorax or tuberculosis (Fig. 13). Pleural calcification is also a common manifestation of asbestos exposure [9]. Empyema necessitans is a rare complication of pleural space infections and occurs when the infected fluid dissects spontaneously into the chest wall from the pleural space [10]. Tuberculosis is more likely than pyogenic organisms to form an empyema necessitans (Fig. 14).

Lymph node calcifications result from healed granulomatous infection, usually tuberculosis or histoplasmosis (Fig. 15). Eggshell calcification is highly suggestive of silicosis or coal workers' pneumoconiosis, but it has also been described in sarcoidosis, Hodgkin's disease and infections such as histoplasmosis or blastomycosis.

\section{Conclusions}

Some high-attenuation pulmonary abnormalities have characteristic CT findings suggesting the correct diagnosis. In other diseases, a combination of clinical features and radiological findings can significantly improve diagnostic accuracy.

\section{References}

1. Marchiori E, Souza AS, Franquet T et al (2005) Diffuse high attenuation pulmonary abnormalities: a pattern-oriented diagnostic approach on high resolution CT. AJR Am J Roentgenol 184:273-282

2. Marchiori E, Franquet T, Gasparetto TD et al (2008) Consolidation with diffuse or focal high attenuation computed tomography findings. J Thorac Imaging 23:298-304

3. Chai JL, Patz EF (1994) CT of the lung: patterns of calcification and other high attenuation abnormalities. AJR Am J Roentgenol 162:1063-1066

4. Marchiori E, Müller NL, Souza AS et al (2005) Unusual manifestations of metastatic pulmonary calcification. J Thorac Imaging 20:66-70

5. Kim KI, Kim CW, Lee MK et al (2001) Imaging of occupational lung disease. Radiographics 21:1371-1391

6. Marchiori E, Ferreira A, Müller NL et al (2001) Silicoproteinosis: high-resolution CT and histologic findings. J Thorac Imaging 16:127-129

7. Brown K, Mund DF, Aberle DR et al (1994) Intrathoracic calcifications: radiographic features and differential diagnosis. Radiographics 14:1247-1261

8. Kanne JP, Godwin JD, Takasugi JE et al (2004) Diffuse pulmonary ossification. J Thorac Imaging 19:98-102

9. Bendayan D, Barziv Y, Kramer MR (2000) Pulmonary calcifications: a review. Respiratory Medicine 94:190-193

10. Peterson MW, Austin M, Yip CK et al (1987) CT findings in transdiaphragmatic empyema necessitatis due to tuberculosis. J Comput Assist Tomogr 11:704-706 